\title{
CONTINUOUS FUNCTIONS ON THE INTERVAL THAT HAVE CONJUGATES NEAR THE IDENTITY
}

\author{
SAM W. YOUNG
}

(Communicated by James E. West)

\begin{abstract}
This paper concerns continuous functions on the unit interval and provides solutions to two problems: Characterize those continuous functions that are topologically equivalent (resp. conjugate) to functions arbitrarily near the identity. The second question was raised by Joe Martin.
\end{abstract}

\section{INTRODUCTION}

The purpose of this paper is to establish characterizations in simple terms of continuous functions that respectively have the following two properties. The letter " $T$ " associates with "topologically equivalent" and " $C$ " with "conjugate."

A continuous function $f:[0,1] \rightarrow[0,1]$ has Property $\mathrm{T}$ iff for each $\varepsilon>0$ there exist homeomorphisms $k, h:[0,1] \rightarrow[0,1]$ such that $|k f h(x)-x|<\varepsilon$ for all $x \in[0,1]$ (or eq. $d(k f h$, id) $<\varepsilon$.)

A continuous function $f:[0,1] \rightarrow[0,1]$ has Property $\mathrm{C}$ iff for each $\varepsilon>0$ there exists a homeomorphism $h:[0,1] \rightarrow[0,1]$ such that $\left|h^{-1} f h(x)-x\right|<\varepsilon$ for all $x \in[0,1]$.

The characterization of property $\mathrm{C}$ answers a question raised by Joe Martin at the Joint Summer Research Conference, Humbolt State University, June 1989 [CTDS, problem 1.2] and stated again at the Spring Topology Conference, Southwest Texas State University, San Marcos, Texas, April, 1990. The arguments in this paper employ nothing more than the elementary properties of continuous functions on the interval. We will find it convenient to abuse the interval notation by allowing $[a, b]=[b, a]=$ the smallest closed interval containing $\{a, b\}$. If $f$ is a continuous function defined on an interval and $f(a)=f(b)$, then $[a, b]$ is called a level set interval (of $f)$. And for any $x$ in the domain of $f,[x, f(x)]$ is called a displacement interval (of $f$ ). Notice that every level set interval is covered by the union of two displacement

Received by the editors October 10,1990; presented to the seventh Auburn Miniconference on Real Analysis, October, 1990, sponsored by N.S.F.

1991 Mathematics Subject Classification. Primary 54C05; Secondary 54H 15.

Key words and phrases. Continuous function, topological equivalence, conjugate, homeomorphism, homotopy. 
intervals; since if $f(a)=f(b)=c$, then $[a, b]=[a, c] \cup[b, c]$. The symbol " $\rightarrow$ " indicates that the function is onto. The linear orientation reversing homeomorphism on $[0,1]$ will be denoted by $J ; J(x)=1-x$.

\section{Property T}

Suppose that $a<b$ and $c<d$. For $x \in[a, b]$, let

$$
x^{\prime}=c+(d-c)(x-a) /(b-a) .
$$

In this section we begin to solve the first problem by establishing

Theorem 1. Suppose $a<b, c<d, f:[a, b] \rightarrow[c, d]$ is continuous, $f^{-1}(c)$ $=\{a\}$, and $\varepsilon>0$. Then there exist homeomorphisms $h:[a, b] \rightarrow[a, b]$ and $k:[c, d] \rightarrow[c, d]$ such that for all $x \in[a, b],\left|k f h(x)-x^{\prime}\right|<\varepsilon$.

Lemma. Suppose $a<b, c<d, f:[a, b] \rightarrow[c, d]$ is continuous, and $f^{-1}(c)$ $=\{a\}$, then there exists a sequence $b=x_{0}>x_{1}>x_{2}>\cdots \rightarrow a$ with the property that $f\left[x_{1}, x_{0}\right] \subset\left(x_{2}^{\prime}, x_{0}^{\prime}\right]$ and $f\left[x_{i+1}, x_{i}\right] \subset\left(x_{i+2}^{\prime}, x_{i-1}^{\prime}\right)$ for $i=1,2,3, \ldots$. Proof of Lemma. We begin with $x_{0}=b$ (thus $x_{0}^{\prime}=d$ ) and choose $x_{1}$ so that $a<x_{1}<\min f^{-1}\left(x_{0}^{\prime}\right)$. Next choose $x_{2}$ so that $x_{2}^{\prime}<\min f\left[x_{1}, x_{0}\right]$ and so that $a<x_{2}<\min \left\{a+\frac{1}{2}, x_{1}, \min f^{-1}\left(x_{1}^{\prime}\right)\right\}$. Now by induction we can choose for all $i \geq 3, x_{i}$ so that $x_{i}^{\prime}<\min f\left[x_{i-1}, x_{i-2}\right]$ and so that $a<x_{i}<\min \left\{a+\frac{1}{i}, x_{i-1}, \min f^{-1}\left(x_{i-1}^{\prime}\right)\right\}$.

The first part of the conclusion is clear since $\min f\left[x_{1}, x_{0}\right]>x_{2}^{\prime}$ and $\max f\left[x_{1}, x_{0}\right] \leq d=x_{0}^{\prime}$. For $i \geq 1$, we have $\min f\left[x_{i+1}, x_{i}\right]>x_{i+2}^{\prime}$. Now if $\max f\left[x_{i+1}, x_{i}\right] \geq x_{i-1}^{\prime}$, then there exists $t \in\left[x_{i+1}, x_{i}\right]$ such that $f(t)=x_{i-1}^{\prime}$. But $x_{i}<\min f^{-1}\left(x_{i-1}^{\prime}\right)$ and so $\max f\left[x_{i+1}, x_{i}\right]<x_{i-1}^{\prime}$. Thus $f\left[x_{i+1}, x_{i}\right] \subset\left(x_{i+2}^{\prime}, x_{i-1}^{\prime}\right)$ and $\left\{x_{i}\right\}$ and $\left\{x_{i}^{\prime}\right\}$ are decreasing sequences converging to $a$ and $c$ respectively.

Proof of Theorem 1. Let $0<r<1$ be such that $(d-c)(1-r)<\varepsilon / 2$. Define $h$ to be an increasing homeomorphism on $[a, b]$ such that $h\left(a+(b-a) r^{i}\right)=x_{i}$ for $i=0,1,2, \ldots$. Define $k$ to be an increasing homeomorphism on $[c, d]$ such that $k\left(x_{i}^{\prime}\right)=\left(a+(b-a) r^{i}\right)^{\prime}$ for $i=0,1,2, \ldots$. The sequences $\left\{x_{i}\right\}$ and $\left\{x_{i}^{\prime}\right\}$ are given by the lemma.

First consider $x \in[a+(b-a) r, b]$. Then $h(x) \in\left[x_{1}, x_{0}\right], f h(x) \in\left(x_{2}^{\prime}, x_{0}^{\prime}\right]$, and $k f h(x) \in\left(\left(a+(b-a) r^{2}\right)^{\prime}, b\right]$. Now suppose $i \geq 1$ and $x \in\left[a+(b-a) r^{i+1}\right.$, $\left.a+(b-a) r^{i}\right]$. Then $h(x) \in\left[x_{i+1}, x_{i}\right], f h(x) \in\left(x_{i+2}^{\prime}, x_{i-1}^{\prime}\right)$, and $k f h(x) \in$ $\left(\left(a+(b-a) r^{i+2}\right)^{\prime},\left(a+(b-a) r^{i-1}\right)^{\prime}\right)$. Of course $k f h(a)=f(a)=c$.

We see that where ever $x$ is located in the partition of $[a, b]$ provided by the sequence $\left\{a+(b-a) r^{i}\right\}$, the image $k f h(x)$ and $x^{\prime}$ are located in the same or in adjacent intervals of the partition of $[c, d]$ provided by the sequence $\left\{\left(a+(b-a) r^{i}\right)^{\prime}\right\}$. Thus the distance from $k f h(x)$ to $x^{\prime}$ in less than twice the length of the longest interval, which is $\left[(a+(b-a) r)^{\prime}, d\right]$. So for all $x \in[a, b]$, $\left|k f h(x)-x^{\prime}\right|<2(d-c)(1-r)<\varepsilon$.

Notice that in the special case $a=c$ and $b=d$, we have $x^{\prime}=x$, the homeomorphism $k$ can be chosen to be $h^{-1}$, and $\left|h^{-1} f h(x)-x\right|<\varepsilon$ for all $x \in[a, b]$. And thus we have 
Corollary. Suppose $a<b, f:[a, b] \rightarrow[a, b]$ is continuous, $f^{-1}(a)=\{a\}$, and $\varepsilon>0$. Then there exists a homeomorphism $h:[a, b] \rightarrow[a, b]$ such that for all $x \in[a, b],\left|h^{-1} f h(x)-x\right|<\varepsilon$.

Theorem 2. Suppose $f:[0,1] \rightarrow[0,1]$ is continuous, then the following are equivalent:

$\mathrm{T}_{1}$. If $\varepsilon>0$, there exist homeomorphisms $k, h:[0,1] \rightarrow[0,1]$ such that $|k f h(x)-x|<\varepsilon$ for all $x \in[0,1]$ (property $\mathrm{T}$ ).

$\mathrm{T}_{2}$. No finite collection of level set intervals of $f$ covers $[0,1]$.

$\mathrm{T}_{3}$. There exists a function $g \in\{f, f J, J f, J f J\}$ and $0 \leq a<1$ such that $g(s) \leq g(a)<g(t)$ for all $0 \leq s \leq a<t \leq 1$.

Property $\mathrm{T}_{3}$ is described this way in order to avoid dealing with four cases of inequalities. If $g=f$, for example, we see that the graph of $f$ is inscribed in two rectangular boxes (the left one is possibly degenerate) that meet at the point $(a, f(a))$. The graph of $f$ is allowed to touch the top of the left box but not the bottom of the right box except at $(a, f(a))$. When we choose $g=f J$, $J f$, or $J f J$, the graph is flipped to the different orientations but still has the special point with respect to the two boxes.

$\mathrm{T}_{2} \Rightarrow \mathrm{T}_{3}$. We begin with the observation that if $f^{-1}(0)$ is connected and contains 0 , then we can choose $g=f$ and $a=\max f^{-1}(0)$ to satisfy $\mathrm{T}_{3}$. Likewise, if $f^{-1}(0)$ is connected and contains 1 , then choose $g=f J$ and $a=\max (f J)^{-1}(0)=\max J f^{-1}(0)$. We see that in any case where $e$ is an end point of $[0,1]$ and $f^{-1}(e)$ is connected and contains an end point of $[0,1]$, we only have to choose the appropriate $g$ and $a$ to obtain condition $\mathrm{T}_{3}$. Thus we can move on to the case where $c=\max f^{-1}(0)>0, d=\min f^{-1}(1)<1$, and $f$ is not constant on either $[0, c]$ or $[d, 1]$. And we do not lose generality by assuming that $c<d$ since otherwise, if $d<c$, we could choose to work with the function $f J$ and have $f J(J(c))=f(c)=0, f J(J(d))=f(d)=1$, and $J(c)<J(d)$.

Let $G$ be the collection of all open intervals $(s, t), s \neq t$ such that $f(s)=$ $f(t)$, or in other words, the interiors of nondegenerate level set intervals of $f$. In the case under consideration, we must have that some element of $G$ contains $c$. This is because $f$ is not identically 0 on $[0, c]$ so that a number $s, 0 \leq s<c$ can be chosen so that $f(s)>0$ and a number $t, c<t \leq 1$ so that $\bar{f}(t)=f(s)$. Similarly, $d$ belongs to an element of $G$. By the same sort of argument, either $[0, c]$ is a level set interval or a subset of an element of $G$.

In light of the above, it follows from the covering theorem that $[c, d]$ is not covered by $G$ and so there exists a number $a, c<a<d$ such that $a$ belongs to no element of $G$. Either $a=\max f^{-1} f(a)$ or $a=\min f^{-1} f(a)$. In the first case, we have $f(a)<f(t)$ for all $t>a$. And $f(s) \leq f(a)$ for all $s \leq a$ since otherwise if $f(s)>f(a)$ for some $s<a$, a number $s^{\prime}$ can be found so that $a<s^{\prime}$ and $f(s)=f\left(s^{\prime}\right)$. Condition $\mathrm{T}_{3}$ has been obtained for $g=f$. The assumption $a=\min f^{-1} f(a)$ leads to $\mathrm{T}_{3}$ for the choice $g=J f J$.

$\mathrm{T}_{1} \Rightarrow \mathrm{T}_{2}$. It suffices to show that if a collection of $n$ level set intervals covers $[0,1]$ and $k$ and $h$ are homeomorphisms, then $d(k f h$, id $) \geq 1 / 2 n$. Suppose that $G$ is such a collection and $k$ and $h$ are homeomorphisms. The collection of intervals that are images under $h^{-1}$ of an element of $G$ also covers $[0,1]$ and at least one such interval has length at least $1 / n$. Thus there exists $x_{1}$, $x_{2} \in[0,1]$ such that $\left|h^{-1}\left(x_{1}\right)-h^{-1}\left(x_{2}\right)\right| \geq 1 / n$ and $f\left(x_{1}\right)=f\left(x_{2}\right)$. 
Now for $x \in[0,1]$,

$$
\begin{aligned}
\max |k f h(x)-x| & \geq \max \left\{\left|k f h h^{-1}\left(x_{1}\right)-h^{-1}\left(x_{1}\right)\right|,\left|k f h h^{-1}\left(x_{2}\right)-h^{-1}\left(x_{2}\right)\right|\right\} \\
& \geq \frac{1}{2}\left|k f h h^{-1}\left(x_{1}\right)-h^{-1}\left(x_{1}\right)\right|+\frac{1}{2}\left|k f h h^{-1}\left(x_{2}\right)-h^{-1}\left(x_{2}\right)\right| \\
& =\frac{1}{2}\left|k f\left(x_{1}\right)-h^{-1}\left(x_{1}\right)+\frac{1}{2}\right| k f\left(x_{1}\right)-h^{-1}\left(x_{2}\right) \mid \\
& \geq \frac{1}{2}\left|h^{-1}\left(x_{1}\right)-h^{-1}\left(x_{2}\right)\right| \geq \frac{1}{2 n} .
\end{aligned}
$$

$\mathrm{T}_{3} \Rightarrow \mathrm{T}_{1}$. Suppose $1>\varepsilon>0$ and that $f$ satisfies $\mathrm{T}_{3}$ for some choice of $g$ and $0 \leq a<1$. If $a=0$ then Theorem 1 obtains $\mathrm{T}_{1}$ directly. Without loss of generality, assume that $f(s) \leq f(a)<f(t)$ for all $0 \leq s \leq a<t \leq 1$ and that $0<a<1$. Otherwise one of the other choices for $g$ does as well.

Let $c=\max \{a, f(a)\}$ and note that $0<c<1$. Let $q:[0,1] \rightarrow[0,1]$ be a homeomorphism with $q(0)=0, q(\varepsilon / 2)=c, q(1)=1$ and $q$ is linear on each of $[0, \varepsilon / 2]$ and $[\varepsilon / 2,1]$. And now consider the conjugate $q^{-1} f q$. The special point $(a, f(a))$ on the graph of $f$ is pushed to the point $\left(q^{-1}(a), q^{-1} f(a)\right)$ on the graph of $q^{-1} f q$ where we have $q^{-1}(a) \leq q^{-1}(c)=\varepsilon / 2$ and $q^{-1} f(a) \leq$ $q^{-1}(c)=\varepsilon / 2$. Whereas $f[0, a]=[0, f(a)]$ we now have $q^{-1} f q\left[0, q^{-1}(a)\right]=$ $\left[0, q^{-1} f(a)\right]$, and whereas $f[a, 1]=[f(a), 1]$ we now have $q^{-1} f q\left[q^{-1}(a), 1\right]$ $=\left[q^{-1} f(a), 1\right]$.

We can now use Theorem 1 to choose homeomorphisms $h:\left[q^{-1}(a), 1\right] \rightarrow$ $\left[q^{-1}(a), 1\right]$ and $k:\left[q^{-1} f(a), 1\right] \rightarrow\left[q^{-1} f(a), 1\right]$ so that $\left|k q^{-1} f q h(x)-x^{\prime}\right|<$ $\varepsilon / 2$ for all $x \in\left[q^{-1}(a), 1\right]$ where $x^{\prime}$ is the linear increasing function of $x$ taking $\left[q^{-1}(a), 1\right]$ onto $\left[q^{-1} f(a), 1\right]$. It is easy to see that $\left|x^{\prime}-x\right|<\varepsilon / 2$ for all $x \in\left[q^{-1}(a), 1\right]$ and thus $\left|k q^{-1} f q h(x)-x\right|<\varepsilon$ for all $x \in\left[q^{-1}(a), 1\right]$.

We can extend $h$ and $k$ respectively to homeomorphisms $\hat{h}$ and $\hat{k}$ on $[0,1]$ by making $\hat{h}$ the identity on $\left[0, q^{-1}(a)\right]$ and $\hat{k}$ the identity on $\left[0, q^{-1} f(a)\right]$. Clearly, $\left|\hat{k} q^{-1} f q \hat{h}(x)-x\right|<\varepsilon$ for $x \in\left[0, q^{-1}(a)\right]$. Now finally, $\left|\hat{k} q^{-1} f q \hat{h}(x)-x\right|<\varepsilon$ for all $x \in[0,1]$.

\section{Property C}

In this section we characterize the functions that have property $\mathrm{C}$. The only distinction is that the special point must be a fixed point. The arguments will be quite similar to those of the preceding section.

Theorem 3. Suppose $f:[0,1] \rightarrow[0,1]$ is continuous. Then the following are equivalent:

$\mathrm{C}_{1}$. If $\varepsilon>0$ there exists a homeomorphism $h:[0,1] \rightarrow[0,1]$ such that $\left|h^{-1} f h(x)-x\right|<\varepsilon$ for all $x \in[0,1]$ (property $\mathrm{C}$ ).

$\mathrm{C}_{2}$. No finite collection if displacement intervals of $f$ covers $[0,1]$.

$\mathrm{C}_{3}$. There exist a function $g \in\{f, J f J\}$ and $0 \leq a<1$ such that $g(a)=a$ and $g(s) \leq g(a)<g(t)$ for all $0 \leq s \leq a<t \leq 1$.

$\mathrm{C}_{2} \Rightarrow \mathrm{C}_{3}$. If $f^{-1}(0)=\{0\}$ then we can choose $g=f$ and $a=0$ and if $f^{-1}(1)=\{1\}$, choose $g=J f J$ and $a=0$. So we have only the case that there exist $c \neq 0$ and $d \neq 1$ such that $f(c)=0$ and $f(d)=1$. It follows that $c<d$ because otherwise $[c, f(c)] \cup[d, f(d)]$ covers $[0,1]$. 
Consider the collection $G$ to which $[s, t]$ belongs iff either $[s, t]$ is a displacement interval or $[s, t]$ is the union of two displacement intervals that intersect only at a common end point. Let $G^{\prime}=\{(s, t):[s, t] \in G\}$, the collection of interiors of elements of $G$. We know that $G^{\prime}$ does not cover $[c, d]$ since in that case there would be a finite subcollection of $G$ covering $[c, d]$ and, therefore, a finite collection of displacement intervals covering $[0,1]$. So let $a \in[c, d]$ be such that $a$ does not belong to any interval of $G^{\prime}$. Observe that $f(a)=a$ since otherwise if $a<f(a)$, for example, then we can find an $x<a$ close enough to $a$ so that $x<a<f(x)$.

Now if there exists $s<a$ such that $f(s)=a$, then there does not exist $t>a$ such that $f(t)=a$ since in this case, $a \in[s, f(s)] \cup[t, f(t)]$ and $f(s)=f(t)=a=[s, f(s)] \cap[t, f(t)]$, which places $a$ in an element of $G^{\prime}$. So it is not possible to have $s<a<t$ such that $f(s)=a=f(t)$, and this condition is equivalent to having either $g=f$ or $g=J f J$ in association with the number $a$.

$\mathrm{C}_{1} \Rightarrow \mathrm{C}_{2}$. It suffices to show that if a collection of $n$ displacement intervals covers $[0,1]$ and $h$ is a homeomorphism, then $d\left(h^{-1} f h, \mathrm{id}\right) \geq 1 / n$. Suppose that $G$ is such a collection and $h$ is a homeomorphism. The collection of intervals that are images under $h^{-1}$ of elements of $G$ also covers $[0,1]$, and at least one such interval must be of length at least $1 / n$. Thus there exists $x_{0} \in[0,1]$ such that $\left|h^{-1}\left(x_{0}\right)-h^{-1} f\left(x_{0}\right)\right| \geq 1 / n$. But $x_{0}=h\left(x_{1}\right)$ for some $x_{1}$ and $\left|h^{-1}\left(x_{0}\right)-h^{-1} f\left(x_{0}\right)\right|=\left|x_{1}-h^{-1} f h\left(x_{1}\right)\right| \geq 1 / n$.

$\mathrm{C}_{3} \Rightarrow \mathrm{C}_{1}$. Let $1>\varepsilon>0$ and assume that $f(a)=a$ and $f(s) \leq f(a)<f(t)$ for all $s \leq a<t$. Otherwise we could choose $g=J f J$. If $a=0$, then the corollary provides a direct proof. If $a>0$, then we define a homeomorphism $q:[0,1] \rightarrow[0,1]$ with $q(0)=0, q(\varepsilon)=a, q(1)=1$ and $q$ is linear on each of $[0, \varepsilon]$ and $[\varepsilon, 1]$. Now $q^{-1} f q[0, \varepsilon]=[0, \varepsilon]$ and $q^{-1} f q[\varepsilon, 1]=[\varepsilon, 1]$. We apply the corollary to obtain to obtain a homeomorphism $h:[\varepsilon, 1] \rightarrow[\varepsilon, 1]$ such that $\left|h^{-1} q^{-1} f q h(x)-x\right|<\varepsilon$ for all $x \in[\varepsilon, 1]$. Then extend $h$ to $\hat{h}$ by making $\hat{h}(x)=x$ for $x \in[0, \varepsilon]$ and $\hat{h}(x)=h(x)$ for $x \in[\varepsilon, 1]$.

Now consider $(q \hat{h})^{-1} f(q \hat{h})=\hat{h}^{-1} q^{-1} f q \hat{h}$. By construction, $\hat{h}^{-1} q^{-1} f q \hat{h}[0, \varepsilon]$ $=[0, \varepsilon]$. If $x \in[\varepsilon, 1]$, then $\hat{h}(x)=h(x)$ and $\left|\hat{h}^{-1} q^{-1} f q \hat{h}(x)-x\right|<\varepsilon$. The homeomorphism $q \hat{h}$ has the desired property.

\section{EXAMPLES AND COMMENTS}

The function $J$ is the simplest example of a function that has property $\mathrm{T}$ but not property $\mathrm{C}$, but this occurs because of a reversal of orientation. The distinction between the two properties is better illustrated by

Example 1. Let $f$ be the function for which $f(0)=\frac{1}{2}, f\left(\frac{1}{4}\right)=0, f\left(\frac{1}{2}\right)=$ $f(1)=1$ and is linear on each of $\left[0, \frac{1}{4}\right],\left[\frac{1}{4}, \frac{1}{2}\right]$, and $\left[\frac{1}{2}, 1\right]$.

The function $f$ does not have any property $C$. Notice that the intervals $\left[0, \frac{1}{2}\right]$, and $\left[\frac{1}{2}, 1\right]$ together constitute a covering of $[0,1]$ by displacement intervals. However $f$ does have property $\mathrm{T}$ since any choice of $a \in\left[\frac{3}{8}, \frac{1}{2}\right]$ provides the special point of condition $\mathrm{T}_{3}$. Also note that $f^{2}(0)=f^{2}(1)=1$ and clearly $f^{2}$ does not have property $\mathrm{T}$. 
Functions with property $\mathrm{T}$ but not property $\mathrm{C}$ can be bound arbitrarily near the identity as demonstrated by the following example, which extends the idea in Example 1.

Example 2. Let $n \geq 3$ be an integer, and let $f$ be the function for which $f(0)=\frac{1}{n}, f\left(\frac{1}{2 n}\right)=0, f\left(\frac{1}{n}\right)=\frac{2}{n}, f\left(\frac{n-1}{n}\right)=f(1)=1$ and $f$ is linear on each of $\left[0, \frac{1}{2 n}\right],\left[\frac{1}{2 n}, \frac{1}{n}\right],\left[\frac{1}{n}, \frac{n-1}{n}\right]$, and $\left[\frac{n-1}{n}, 1\right]$.

The intervals $\left[0, \frac{1}{n}\right],\left[\frac{1}{n}, \frac{2}{n}\right],\left[\frac{2}{n}, \frac{3}{n}\right], \ldots,\left[\frac{n-1}{n}, 1\right]$ provide a covering by displacement intervals. On the other hand, any choice of $a \in\left[\frac{3}{4 n}, \frac{n-1}{n}\right]$ provides a special point. Thus $f$ has property $\mathrm{T}$ but not property $\mathrm{C}$. It is clear that $d(f$, id $)=\frac{1}{n}$, but furthermore, no conjugate of $f$ is any closer to the identity. See the argument for $C_{1} \Rightarrow C_{2}$.

In the proof of Theorem 1 , the interval $[a, b]$ was partitioned by the geometric sequence $\left\{a+(b-a) r^{i}\right\}$. Any partition of sufficiently small mesh provided by a decreasing sequence converging to $a$ would do as well. But the number $r$, $\frac{1}{2} \leq r<1$, serves as a useful parameter. It allows us to construct a homotopy from a suitable function $f$ to the identity that preserves property $\mathrm{T}$ or property $\mathrm{C}$, respectively, for $r<1$. Also, the special point can be continuously pushed to an end point as is done in the construction in $T_{3} \Rightarrow T_{1}$ and $C_{3} \Rightarrow C_{1}$. Thus we could add the following to the statements of Theorems 2 , and 3 , respectively.

$\mathrm{T}_{4}$. There exists a homotopy $H:[0,1] \times[0,1] \rightarrow[0,1]$ such that $H(\cdot, 0)=$ $f, H(\cdot, r)$ is topologically equivalent to $f$ for $0 \leq r<1$, and $H(\cdot, 1)=\mathrm{id}$.

$\mathrm{C}_{4}$. There exists a homotopy $H:[0,1] \times[0,1] \rightarrow[0,1]$ such that $H(\cdot, 0)=$ $f, H(\cdot, r)$ is conjugate to $f$ for $0 \leq r<1$ and $H(\cdot, 1)=\mathrm{id}$.

\section{REFERENCES}

[CTDS] Morton Brown, ed., Continuum theory and dynamical systems, Contemp. Math., vol. 117, Amer. Math. Soc., Providence, RI, 1991.

Department of Mathematics, Auburn University, Auburn, Alabama $36849-5310$

E-mail address: SWYOUNG@DUCVAX.AUBURN.EDU 